\title{
Percepción de los alumnos sobre su formación profesional como Licenciados en Gestión Tecnológica
}

\section{Student's perception of their professional training as Graduates in Technology Management}

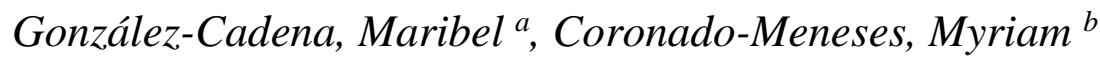

\begin{abstract}
:
This research, which identifies the perception of the students of the Bachelor's Degree in Technology Management, of the Higher School of Tizayuca, regarding the programmatic contents during their formation, it is constituted with the analysis of information in two stages.

In the first stage, research was carried out in academic documents and official pages of the Autonomous University of the State of Hidalgo regarding the subject programs that, during their training, the student takes. In the second stage, a research instrument was designed and primary information is presented based on the analysis of the results of its application to students enrolled in the second to ninth semester of the educational program.

The research results show that the students identify congruence between the contents of the educational program and the activities that they will carry out as a graduate of the Bachelor of Technology Management.

Regarding the opinion of subjects per semester, they consider that the learning of Fundamentals of Management and Technology Watch, Technology-Based Business Administration, Management and Technology Plan, Regulatory Framework for Science and Technology, Commercialization of Technology Projects and Quality Management, Protection and Intellectual Capital and Financial Management of Technological Projects, are relevant for their professional practice; however, they ignore the formation of Professional Ethics and Cause and Effect. Foreign language
\end{abstract}

Keywords:

Technology Management, Graduation Profile, Vocational Training.

\section{Resumen:}

La presente investigación, que identifica la percepción de los alumnos de la Licenciatura en Gestión Tecnológica, de la Escuela Superior de Tizayuca, respecto a los contenidos programáticos durante su formación, se constituye con el análisis de información en dos etapas.

En la primera etapa se realizó investigación en documentos académicos y páginas oficiales de la Universidad Autónoma del Estado de Hidalgo respecto a los programas de asignatura que, durante su formación, cursa el alumno. En la segunda etapa se diseñó un instrumento de investigación y se presenta información primaria con base al análisis de resultados de su aplicación a los alumnos matriculados de segundo a noveno semestre del programa educativo.

Los resultados de investigación exhiben que los alumnos identifican congruencia entre los contenidos del programa educativo y las actividades que realizará como egresado de la Licenciatura en Gestión Tecnológica.

Respecto a la opinión de asignaturas por semestre, consideran que el aprendizaje de Fundamentos de Gestión y Vigilancia Tecnológica, Administración de Empresas de Base Tecnológica, Gestión y Plan Tecnológico, Marco Regulatorio para la Ciencia y Tecnología, Comercialización de Proyectos Tecnológicos y Gestión de la Calidad, Protección y Capital Intelectual y Administración Financiera de Proyectos Tecnológicos, son relevantes para su ejercicio profesional; sin embargo, soslayan la formación de Ética Profesional y de Causa y Efecto. Lengua Extranjera.

\section{Palabras Clave:}

\footnotetext{
a Maribel González-Cadena, Universidad Autónoma del Estado de Hidalgo, Escuela Superior de Tizayuca; https://orcid.org/0000-0001-53710442, e-mail: maribel_gonzalez4257@uaeh.edu.mx

b Myriam Coronado Meneses, Universidad Autónoma del Estado de Hidalgo, Escuela Superior de Tizayuca, https://orcid.org/0000-00031171-516X, Email: myriam_coronado3187@uaeh.edu.mx
} 


\section{Introducción}

El presente estudio tiene como propósito realizar una investigación por medio de la recopilación de datos que coadyuven a identificar las incidencias, desde la perspectiva de los alumnos, al cursar el programa educativo de la Licenciatura en Gestión Tecnológica de la Universidad Autónoma del Estado de Hidalgo.

Para la realización de este estudio se emplea un cuestionario con preguntas cerradas; los elementos que se consideraron fueron: contenidos programáticos, secuencia y utilidad, entre otros.

El instrumento fue aplicado a 67 estudiantes inscritos desde segundo hasta octavo semestre; los cuales respondían lo concerniente al periodo educativo inmediato anterior cursado.

\section{Objetivo General}

Identificar la percepción de los alumnos sobre las competencias que desarrollaron durante su formación como Licenciados en Gestión Tecnológica de la Escuela Superior de Tizayuca.

\section{Materiales y Métodos}

Para cumplir con el objetivo de este trabajo, la investigación ostentó un diseño no experimental de tipo transversal con alcance descriptivo.

\section{Resultados Generales del Programa Educativo}

Con relación al ítem acerca de las razones de elección de la licenciatura, los alumnos manifestaron, con mayor frecuencia de contestación (74\%), que las principales razones que lo llevaron a elegir como carrera la Licenciatura en Gestión Tecnológica son las habilidades en el área (29\%), la cercanía domiciliar de la institución (26\%) y por la recomendación de amigos o familiares (19\%). Por lo tanto, es evidente que los estudiantes eligen a la licenciatura por la vocación y las ventajas que representa contar con una institución de educación superior cercana a su lugar de origen.

En cuanto a la comodidad con la carrera que estudian, se tienen los siguientes resultados: el $97 \%$ de respuestas son favorables, debido a que los estudiantes expresan sentirse identificados con el programa educativo y se encuentran motivados por aprender contenidos disciplinares.
Otro aspecto que ha sido contestado por los alumnos son las actividades relacionadas de un egresado de la Licenciatura en Gestión Tecnológica. Con base en los resultados del instrumento se observa que el $45 \%$ son actividades afines en la integración, evaluación, diseño de metodologías y modelos de gestión del conocimiento y empresarial; de igual forma, los sistemas de evaluación y gestión del Capital Intelectual. Con un 10\% los alumnos mencionan que al egresar se dedicaran a la formación de empresas de base tecnológica.

\section{Opinión descriptiva por semestre/asignatura}

\section{Primer semestre}

Las asignaturas que resultan muy interesantes para los alumnos en primer semestre fueron Administración de Empresas de Base Tecnológica, Conversaciones Introductorias. Lengua Extranjera y Fundamentos de Gestión y Vigilancia Tecnológica, debido a que estas asignaturas son base para el desarrollo de su profesión.

Con relación a la cantidad de temas por asignatura, se exhibe que, de siete asignaturas, cinco son suficientes y los dos restantes los estudiantes manifiestan que son demasiados temas los que se ven en el semestre.

En cuanto a la utilidad profesional del contenido por asignatura, los alumnos consideran que todas son necesarias para ellos en el futuro desempeño laboral ya que, encuentran conocimiento y habilidades que se abordan que les servirá en el ejercicio de su profesión.

\section{Segundo semestre}

Con relación al interés en las asignaturas, los resultados revelan que todas sus asignaturas son interesantes; no obstante, el $50 \%$ de las mismas las consideran muy interesantes (Gestión y Plan Tecnológico, Contabilidad Básica y Marco Regulatorio para la Ciencia y Tecnología). Por lo que respecta a la cantidad de temas por materia, los estudiantes exhiben que la asignatura que tiene demasiados temas es Marco Regulatorio para la Ciencia y Tecnología, caso contrario para el resto.

Los resultados para el ítem vinculado a la utilidad profesional del contenido por asignatura en el segundo semestre, se considera que el $100 \%$ de éstas son necesarias para ellos en el futuro desempeño laboral. 


\section{Tercer semestre}

Con relación al interés que muestran los alumnos al cursar las asignaturas de este ciclo se ostenta que, Mercadotecnia y Comercialización de Proyectos Tecnológicos y Gestión de la Calidad y su Articulación al Desarrollo Tecnológico son muy interesantes y el resto solo son interesantes para los estudiantes.

De acuerdo a la cantidad de temas abordados en cada una de las asignaturas, los estudiantes coinciden en que son suficientes en todas; sin embargo, en Economía de la Empresas, los alumnos exhiben que son pocos temas vistos.

Finalmente, para la utilidad profesional del contenido por asignatura, el 100\% de éstas son necesarias para ellos en el futuro desempeño laboral.

\section{Cuarto semestre}

Las respuestas relacionadas por el interés por cursar las asignaturas de este período escolar reflejan que, para todas las materias existe motivación e interés por su estudio; sin embargo, en Protección y Capital Intelectual y Administración Financiera de Proyectos Tecnológicos son las que muestran un mayor interés.

La cantidad de temas por materia muestra que la asignatura de Decisiones Personales. Lengua Extranjera son pocos, lo cual los alumnos comentan que es necesario incluir más temas en ésta. Caso contrario para las asignaturas de Protección y Capital Intelectual y Administración Financiera de Proyectos Tecnológicos, donde los alumnos manifiestan que son demasiados temas en ambas.

Los resultados del ítem acerca de la vinculación de la utilidad profesional del contenido por asignatura, los alumnos revelan que todas las asignaturas del cuarto semestre son necesarias para ellos en el futuro desempeño laboral.

\section{Quinto semestre}

La mayoría de las materias de quinto semestre existe interés por su estudio; pero, la asignatura de Causa y Efecto. Lengua Extranjera, los estudiantes la consideran nada interesante, por lo cual es importante analizar porque los alumnos la consideran así con la finalidad de establecer estrategias que permitan cambiar esta percepción.
La cantidad de temas por materia muestra que, para la asignatura de Causa y Efecto. Lengua Extranjera son muy poco; en caso contrario se encuentra la asignatura de Implantación Tecnológica que con base en la opinión de los estudiantes son demasiados temas.

En cuanto a la vinculación con la utilidad profesional del contenido por asignatura, para los alumnos señalan que todas son necesarias para ellos en el futuro desempeño laboral.

\section{Sexto semestre}

Los alumnos señalan que, en el $80 \%$ de las asignaturas de este semestre son interesantes; sin embargo, en Ética Profesional manifiestan que no es interesante conocer la importancia de la ética en el desarrollo de la profesión.

Los alumnos comentan que la cantidad de temas vistos en clases son suficientes; caso contrario para la asignatura de la Optativa II. Logística y Cadena de Suministro de Valor que, de acuerdo con la percepción de alumnos son demasiados.

Para los resultados del ítem relativo a la utilidad profesional del contenido por asignatura, se muestra que, en la mayoría de sus materias son necesarias para ellos en el futuro desempeño laboral.

\section{Séptimo semestre}

Todas las asignaturas del séptimo semestre existen interés por su estudio; la cantidad de temas vistos son suficientes para cumplir con los objetivos esperados $\mathrm{E}$ igualmente, el $100 \%$ de éstas tienen utilidad profesional del contenido por asignatura.

\section{Octavo semestre}

Para el ítem relacionado al interés por cursar las asignaturas en este período escolar, se refleja que, para la mayoría de éstas existe interés por su estudio.

En cuanto a la cantidad de temas por materia se obtiene que para todas las asignaturas son suficientes para cumplir con los objetivos establecidos.

Finalmente, con relación a la utilidad profesional del contenido de la asignatura, los alumnos de octavo semestre suponen que la mayoría de éstas tienen mucha utilidad debido a que, son materias disciplinares.

\section{Conclusiones}


Con base en los resultados obtenidos se concluye lo siguiente:

Los resultados son favorables en el conocimiento del plan de estudios de la licenciatura debido a que, el $94.5 \%$ refieren tener ideas de este, lo cual posibilita que tengan claro la trayectoria académica a seguir.

Existe correspondencia entre lo que establece el plan de estudios y las actividades que realiza un egresado de la Licenciatura en Gestión Tecnológica destacándose en la integración, evaluación, diseño de metodologías y modelos de gestión del conocimiento y empresarial; así como, de sistemas de evaluación y gestión del Capital Intelectual para diversas organizaciones. En una menor proporción, los estudiantes señalan que, pueden dedicarse en la implementación de propuestas de solución a problemas relacionados con la gestión de la tecnología, innovación y gestión del conocimiento y/o, a la promoción de formación de empresas de base tecnológicas.

Las asignaturas relacionadas a la 'Transferencia Tecnológica y Gestión de la Calidad y su Articulación con el Desarrollo Tecnológico son básicas para la formación disciplinar.

Caso contrario, con base en la percepción de los alumnos, las asignaturas de idioma inglés no son indispensables dentro de su formación.

\section{Referencias}

[1] UAEH. (2009). Programa Educativo de Licenciatura en Gestión Tecnológica. Documento de Diseño Curricular

[2] Ponce de León, María del Socorro (2002) Guía para realizar estudios de factibilidad y pertinencia de programas educativos. Dirección General de Planeación. Universidad Autónoma del Estado de Hidalgo. 